\title{
Centralização da Circulação Fetal em Gestações de Alto Risco: Avaliação da Vitalidade Fetal e Resultados Perinatais
}

\author{
Redistribution of Fetal Circulation: Assessment of Fetal Well-Being and Perinatal \\ Outcome in High Risk Pregnancies
}

Roseli Mieko Yamamoto Nomura, Rossana Pulcineli Vieira Francisco, Kathia Sakamoto Seizo Miyadahira, Marcelo Zugaib

\section{RESUMO}

Objetivos: analisar, nas gestações de alto risco com diagnóstico de centralização da circulação fetal, os resultados perinatais e a avaliação da vitalidade fetal de acordo com a dopplervelocimetria da artéria umbilical (AU).

Métodos: foram estudadas 717 gestantes de alto risco avaliadas pelo Setor de Vitalidade Fetal da Clínica Obstétrica do HC-FMUSP. Todas foram submetidas aos exames em periodos de até 72 horas que antecederam o parto. Foram excluidas as gestações múltiplas e as com diagnóstico de malformações fetais. A centralização foi caracterizada de acordo com o valor do indice de pulsatilidade (IP) da ACM (IP abaixo do 5o percentil para a idade gestacional = fetos com centralização). A dopplervelocimetria da AU foi classificada como alterada quando a relação $A / B$ foi superior ao percentil 95 para a idade gestacional.

Resultados: nas gestantes com dopplervelocimetria da AU normal (560 pacientes - 78,1\%), observamos correlação apenas entre a centralização e a cardiotocografia suspeita ou alterada (17,1\%). Nas gestantes com dopplervelocimetria da AU alterada (157 pacientes - 21,9\%) as médias dos seguintes parâmetros foram significativamente menores no grupo com

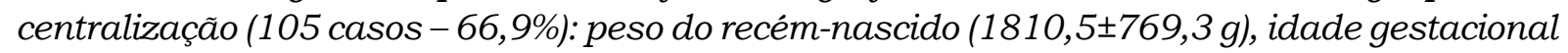
$(34,4 \pm 3,6 \mathrm{sem})$ e $\mathrm{pH}$ no nascimento $(7,20 \pm 0,1)$. Houve também, neste grupo, correlação significativa entre a centralização e alterações na cardiotocografia (57,2\%), indice de Apgar de $1^{\circ}$ minuto inferior a $7(43,8 \%)$ e $5^{\circ}$ minuto inferior a $7(12,4 \%)$.

Conclusões: o diagnóstico da centralização da circulação fetal pela dopplervelocimetria da ACM é significativo apenas em gestações que cursam com algum grau de insuficiência placentária, não se relacionando com piora dos resultados perinatais em gestantes com função placentária normal.

PALAVRAS-CHAVE: Dopplervelocimetria. Morbidade perinatal. Vitalidade fetal.

\section{Introdução}

As provas de avaliação da vitalidade fetal são indicadas em diversos estados que implicam riscos na deterioração da oxigenação do produto

Setor de Vitalidade Fetal

Clínica Obstétrica - Hospital das Clínicas

Faculdade de Medicina da Universidade de São Paulo

Correspondência:

Roseli Mieko Yamamoto Nomura

Rua General Canavarro, 280 - Bairro Campestre

09070-440 - Santo André - SP

Tel: (11) 4991-2481/ 4221-8778; FAX: (11) 4221-8752

e-mail: roselinomura@uol.com.br / vitalidade@hcnet.usp.br

Home Page: http://www.hcnet.usp.br/ob/ conceptual. Neste contexto, a dopplervelocimetria tem sido amplamente utilizada em Obstetricia, principalmente no estudo da função placentária, pela análise dos sonogramas do cordão umbili$\mathrm{cal}^{1,2}$.

A função placentária deficiente, dificultando as trocas gasosas entre o organismo materno e o fetal, é responsável pelo prejuízo na oxigenação fetal. O mecanismo pelo qual o feto se adapta diante dos distúrbios de sua oxigenação são estudados há longa data. Em trabalhos experimentais foram descritas alterações hemodinâmicas fetais como resposta à hipoxia ${ }^{3,4}$, que culminam com ocorrência da acidose. Behrman et al. ${ }^{5}$ demons- 
traram que, em fetos de primata, após hipoxia, existe distribuição preferencial do fluxo sangüineo para órgãos nobres como cérebro, coração e adrenais, com diminuição do fluxo para rins e pulmões. Relataram também que a redistribuição do sangue, inicialmente, é decorrente do estímulo de quimiorreceptores situados na aorta fetal, determinando a vasoconstricção periférica, responsável pelo aumento da resistência vascular periférica. No sistema nervoso central, ocorre vasodilatação e aumento da perfusão tecidual.

As alterações hemodinâmicas da circulação fetal podem ser avaliadas por meio da tecnologia da dopplervelocimetria. Tal como demonstrado nos estudos experimentais, os fetos humanos também apresentam alterações na circulação com redistribuição do débito cardíaco durante o processo de hipoxia ${ }^{6-8}$. Neste fenômeno adaptativo conhecido como centralização da circulação fetal ou "brain-sparing effect", observa-se aumento da velocidade diastólica no fluxo sangüíneo das artérias cerebrais, conseqüente à vasodilatação. Clinicamente este evento corresponde à primeira alteração provocada pela hipoxemia fetal.

Observações realizadas nesta instituição e em outros serviços sugerem que em várias situações clínicas, que podem variar da simples hipotensão materna até a insuficiência placentária, a centralização da circulação fetal é freqüentemente diagnosticada. Este evento relaciona-se à hipoxemia fetal e geralmente precede o desenvolvimento da hipoxia e acidemia nos casos de insuficiência placentária. Estudos longitudinais relatam intervalo médio de duas semanas entre a centralização da circulação fetal e a ocorrência de desacelerações tardias na cardiotocografia $(\mathrm{CTG})^{6}$.

De acordo com as recomendações do "American College of Obstetricians and Gynecologists", a incorporação da dopplervelocimetria da artéria cerebral média (ACM) fetal na prática clínica pode promover intervenções desnecessárias na gestação, devendo portanto ser considerado apenas um exame investigacional dentro da propedêutica fetal. Deste modo, tornase importante o estudo da centralização da circulação fetal e o prognóstico da gestação, quando se caracteriza esta situação.

Este estudo tem como objetivo analisar, nas gestações de alto risco, a correlação entre o diagnóstico de centralização da circulação fetal pela avaliação da dopplervelocimetria da ACM e os resultados perinatais, e a avaliação da vitalidade fetal, levando em consideração os resultados da dopplervelocimetria da artéria umbilical (AU).

\section{Pacientes e Métodos}

Este trabalho foi desenvolvido no Setor de Vitalidade Fetal da Clínica Obstétrica do Hospital das Clínicas da Faculdade de Medicina da Universidade de São Paulo (HC-FMUSP). A população foi selecionada retrospectivamente a partir da consulta do banco de dados do Setor, entre as gestantes atendidas no período compreendido entre janeiro de 1992 e junho de 1998. Foram excluídas as gestações gemelares, as com anomalias fetais e as pacientes cujo parto não foi realizado nesta instituição. Este estudo foi aprovado pela Comissão de Ética para Análise de Projetos de Pesquisa (CAPEPesq) da Diretoria Clínica do HC-FMUSP.

As características das gestantes em relação à idade, paridade e cor estão demonstradas na Tabela 1. Destacamos o predomínio da cor branca, com média de idade materna e paridade não diferindo significativamente entre os grupos analisados. Em relação aos outros diagnósticos clínicos e/ou obstétricos associados, observamos que as sindromes hipertensivas se destacam como a intercorrência mais freqüente, presente em $43,8 \%$ (314 casos). Outras intercorrências associadas incluem o diabete (98 casos - 13,7\%), a suspeita de restrição do crescimento fetal (93 casos $12,9 \%$ ) e as cardiopatias (91 casos - 12,7\%).

Tabela 1 - Principais características das pacientes de acordo com o resultado da dopplervelocimetria da artéria umbilical, segundo o diagnóstico ou não de centralização fetal.

\begin{tabular}{|c|c|c|c|c|c|c|}
\hline \multirow[b]{2}{*}{ Características } & \multicolumn{3}{|c|}{ Artéria Umbilical Normal } & \multicolumn{3}{|c|}{ Artéria Umbilical Alterada } \\
\hline & $\begin{array}{l}\text { ACM Normal } \\
(n=373)\end{array}$ & $\begin{array}{c}\text { ACM Alterada } \\
\text { (Centralização } n=187 \text { ) }\end{array}$ & $p$ & $\begin{array}{c}\text { ACM Normal } \\
\quad(n=52)\end{array}$ & $\begin{array}{c}\text { ACM Alterada } \\
\text { (Centralização } n=105 \text { ) }\end{array}$ & $p$ \\
\hline Idade materna em anos - média (DP) & $29,9(6,9)$ & $27,8(6,7)$ & NS & $27,4(6,9)$ & $29,7(6,8)$ & NS \\
\hline Paridade - média (DP) & $2,7(1,9)$ & $2,6(1,9)$ & NS & $2,9(2,4)$ & $2,8(1,9)$ & NS \\
\hline Cor branca - $\mathrm{N}^{0}$. de casos(\%) & $185(49,6 \%)$ & $77(41,2 \%)$ & NS & $29(55,8 \%)$ & $62(59,1 \%)$ & NS \\
\hline
\end{tabular}

$\mathrm{DP}=$ Desvio-padrão, NS = não-significativo, $\mathrm{ACM}$ = artéria cerebral média.

Um total de 717 gestantes de alto risco fo- ram submetidas a estudo dopplervelocimétrico das 
artérias umbilicais e cerebral média no Setor de Vitalidade Fetal, no máximo 72 horas antes do momento do parto. A dopplervelocimetria foi realizada por via transabdominal, utilizando-se dos seguintes equipamentos de ultra-sonografia: ATL Ultramark 9 com Doppler colorido e Diasonics SPA1000 com Doppler pulsátil. Os exames foram realizados com a paciente em posição semi-sentada. Os sonogramas dos vasos foram obtidos durante a inatividade fetal e em períodos de apnéia. Foram utilizados filtros de baixa freqüência (50 hertz) e analisados sonogramas com ondas uniformes.

O sonograma das AU foi obtido por meio da insonação em alça livre de cordão. Quando evidenciadas velocidades diastólicas reduzidas, procurou-se analisar o cordão na porção próxima à inserção placentária. Foi calculada a relação sístole/diástole (A/B) e o resultado foi classificado como alterado quando superior ao percentil $95 \mathrm{da}$ curva de normalidade adotada ${ }^{10}$ neste Setor.

A dopplervelocimetria da ACM foi realizada em corte transverso do polo cefálico fetal, na altura dos tálamos, com o transdutor deslocado obliquamente em direção à base do crânio. Neste ponto a artéria é facilmente identificada dirigindo-se ântero-lateralmente. Evitou-se a compressão do abdome materno, uma vez que a compressão do polo cefálico fetal pode levar a alterações nos exames realizados nas artérias intracranianas. Para o diagnóstico de centralização da circulação fetal, os resultados foram classificados como alterados (fetos centralizados) quando o valor do índice de pulsatilidade estivesse abaixo do percentil 5 da curva de normalidade de Arduini e Rizzo ${ }^{11}$, em idade gestacional correspondente. O diagnóstico de centralização fetal não influenciou na conduta clínica, nem foi utilizado como critério para resolução da gestação.

A CTG anteparto de repouso foi realizada por um periodo mínimo de 15 minutos, com a paciente em posição de semi-Fowler. Utilizaram-se de aparelhos da marca Hewlett Packard, modelos HP $8041 \mathrm{~A}$, com velocidade de registro gráfico de um centímetro por minuto. De acordo com o índice cardiotocométrico de Zugaib e Behle ${ }^{12}$, o feto foi classificado como ativo (vitalidade normal), hipoativo (vitalidade suspeita) ou inativo (vitalidade alterada). Em face de um resultado suspeito ou alterado, realizou-se o teste da estimulação sônica ${ }^{12}$. O feto foi então classificado como reativo (aceleração da freqüência cardíaca fetal - FCF de $20 \mathrm{bpm}$ por 3 minutos), hiporreativo (aceleração da FCF inferior a $20 \mathrm{bpm}$ ou a 3 minutos) ou inativo (ausência de aceleração da FCF). A resposta fetal foi classificada também como padrão bifásico ou monofásico, de acordo com a constatação ou não de acelerações transitórias após o término da resposta da FCF à estimulação sônica. De acordo com os critérios adotados neste Setor, a CTG foi classificada como normal, quando o feto foi ativo ou reativo, ou com padrão bifásico; suspeita quando hipoativo e hiporreativo, mantendo padrão monofásico, ou alterada, quando inativo e não-reativo.

O perfil biofisico fetal $(\mathrm{PBF})$ incluiu a análise das atividades biofisicas fetais: a FCF, analisada pela CTG, movimentos respiratórios fetais, movimentos corpóreos fetais, tônus fetal e a avaliação do volume de líquido amniótico. Os parâmetros biofísicos foram avaliados com aparelho de ultra-sonografia da marca Toshiba SAL77B ou Diasonics SPA 1000. Durante o período máximo padronizado para observação das atividades biofísicas (30 minutos), foi caracterizada como normal a ocorrência de no mínimo um episódio de movimento respiratório com duração mínima de 30 segundos. Os movimentos corpóreos fetais foram classificados como normais quando o feto apresentou no mínimo um movimento amplo ou três movimentos lentos durante o período de observação, e o tônus como normal na presença de movimentos corpóreos fetais ou de acordo com os movimentos de abertura e fechamento das mãos. O volume de líquido amniótico foi analisado pela medida do índice de líquido amniótico (ILA), calculado de acordo com a metodologia proposta por Phelan et al., ${ }^{13}$, e o oligoidrâmnio foi caracterizado quando ILA $\leq 5,0 \mathrm{~cm}$, sendo este limite utilizado na caracterização do volume de líquido amniótico anormal dentro do PBF. Cada parâmetro do PBF recebeu o escore 2 quando normal e o escore 0 quando anormal, sendo classificado de acordo com o seu somatório em: normal (8 ou 10), suspeito (6) ou alterado (4, 2 ou 0$)$.

Foram analisados os seguintes resultados neonatais: idade gestacional no nascimento, indices de Apgar de 1ㅇ e 5o minutos, $\mathrm{pH}$ da artéria umbilical no nascimento, peso do recém-nascido (RN), adequação do peso do RN à idade gestacional e mortalidade perinatal. Os RN foram classificados como pequeno para a idade gestacional, quando com peso inferior ao percentil 10 para a idade gestacional, de acordo com a curva de normalidade desenvolvida pelo setor de neonatologia deste hospital ${ }^{14}$.

A idade gestacional foi calculada a partir da data da última menstruação (DUM) quando esta foi compativel com exame ultra-sonográfico realizado até a 20 a semana. Quando a paciente não sabia referir a DUM ou na discordância com o exame ultra-sonográfico, a datação da gestação foi realizada com base na primeira ultra-sonografia.

Foram analisadas as freqüências dos resul- 
tados dos exames para comparação entre os grupos de estudo. Para as variáveis qualitativas utilizou-se do teste de $\chi^{2}$ com correção de Yates para continuidade e, quando pertinente, o teste exato de Fisher. Para as variáveis quantitativas, utilizou-se do teste $t$ de Student. Adotou-se como nivel de significância o valor $0,05(\alpha=5 \%)$. Com isso, niveis descritivos (p) inferiores a esse valor foram considerados significantes $(p<0,05)$.

\section{Resultados}

Foram analisadas no presente trabalho setecentos e dezessete gestantes de alto risco; a dopplervelocimetria das AU mostrou-se normal em 560 casos $(78,1 \%)$, e neste grupo a centralização da circulação fetal foi caracterizada em $187(33,4 \%)$ casos. O exame dopplervelocimétrico das AU mostrou-se alterado em 157 gestantes $(21,9 \%)$, e a centralização foi caracterizada em proporção significativamente $(\mathrm{p}<0,0001)$ maior de pacientes (105 casos $-66,9 \%)$.
A distribuição da população segundo os resultados dos testes de avaliação da vitalidade fetal de acordo com os resultados da dopplervelocimetria está demonstrada na Tabela 2.

Nas gestações com dopplervelocimetria normal das AU, a centralização da circulação fetal correlacionou-se significativamente somente com as alterações na CTG anteparto de repouso. Não foram constatadas diferenças significativas na distribuição dos resultados do PBF de acordo com a ocorrência ou não da centralização fetal. Em relação ao ILA, podemos notar elevada proporção de oligoidrâmnio, porém com distribuição semelhante nos grupos analisados, sem diferenças estatisticamente significativas.

Nos casos com dopplervelocimetria alterada da AU (Tabela 2), a proporção de CTG anormais foi significativamente superior nas gestações com centralização fetal. Do mesmo modo, a ocorrência de PBF inferior a 6 apresentou associação significativa com a centralização fetal. Na análise do ILA, não foi demonstrada correlação estatística entre a redistribuição da circulação fetal e o diagnóstico de oligoidrâmnio.

Tabela 2 - Distribuição da população segundo os resultados dos testes de avaliação da vitalidade fetal de acordo com o resultado da dopplervelocimetria da artéria umbilical e da artéria cerebral média.

\begin{tabular}{|c|c|c|c|c|c|c|}
\hline \multirow[b]{2}{*}{$\begin{array}{l}\text { Avaliação da } \\
\text { Vitalidade Fetal }\end{array}$} & \multicolumn{3}{|c|}{ Artéria Umbilical Normal } & \multicolumn{3}{|c|}{ Artéria Umbilical Alterada } \\
\hline & $\begin{array}{l}\text { ACM Normal } \\
\qquad(\mathrm{n}=373)\end{array}$ & $\begin{array}{c}\text { ACM Alterada } \\
\text { (Centralização } n=187 \text { ) }\end{array}$ & $p$ & $\begin{array}{l}\text { ACM Normal } \\
(n=52)\end{array}$ & $\begin{array}{c}\text { ACM Alterada } \\
\text { (Centralização } n=105 \text { ) }\end{array}$ & $p$ \\
\hline \multicolumn{7}{|l|}{ Cardiotocografia } \\
\hline Normal & $345(92,5)$ & $155(82,9)$ & \multirow{3}{*}{$<0,005$} & $40(76,9)$ & $45(42,9)$ & \multirow{3}{*}{$<0,0005$} \\
\hline Suspeita & $20(5,4)$ & $25(13,4)$ & & $6(11,5)$ & $30(28,6)$ & \\
\hline Alterada & $8(2,1)$ & $7(3,7)$ & & $6(11,5)$ & $30(28,6)$ & \\
\hline \multicolumn{7}{|l|}{ Perfil biofísico fetal } \\
\hline $8-10$ & $334(89,5)$ & $168(89,8)$ & \multirow{2}{*}{ NS } & $44(84,6)$ & $58(55,2)$ & \multirow{2}{*}{$<0,001$} \\
\hline$\leq 6$ & $39(10,5)$ & $19(10,2)$ & & $8(15,4)$ & $47(44,8)$ & \\
\hline \multicolumn{7}{|c|}{ Índice de líquido amniótico } \\
\hline$>5,0 \mathrm{~cm}$ & $243(65,2)$ & $122(65,3)$ & \multirow{2}{*}{ NS } & $34(65,4)$ & $66(62,9)$ & \multirow{2}{*}{ NS } \\
\hline$\leq 5,0 \mathrm{~cm}$ & $130(34,8)$ & $65(34,7)$ & & $18(34,6)$ & $39(37,1)$ & \\
\hline
\end{tabular}

NS = Não-significativo, ACM = artéria cerebral média.

A análise dos resultados perinatais (Tabela 3) demonstra que, nos casos com resultado normal na dopplervelocimetria da artéria umbilical, não foi constatada nenhuma correlação estatisticamente significativa entre o diagnóstico de centralização da circulação fetal e resultados adversos.

A análise dos casos com dopplervelocimetria alterada das AU demonstrou associação estatisticamente significativa entre a centralização da circulação fetal e os resultados perinatais alterados (Tabela 3). No grupo que apresentou centralização as médias da idade gestacional, do peso dos recém nascidos e do $\mathrm{pH}$ da $\mathrm{AU}$ no nascimento foram significativamente menores que no grupo com dopplervelocimetria da ACM normal. Apesar de não ter sido realizada a gasometria do cordão umbilical na totalidade dos casos e da diferença observada na média entre os grupos, foi possivel constatar que a proporção de acidose no nascimen- 
to ( $\mathrm{pH}$ inferior a 7,20$)$ não foi significativamente maior no grupo com centralização. A ocorrência de casos com índices de Apgar de 1ํ e 5은 minutos inferiores a 7 foi significativamente maior no grupo centralizado, porém, não foram constatadas diferenças significativas na mortalidade perinatal.

Tabela 3 - Distribuição da população segundo os resultados perinatais e de acordo com o resultado da dopplervelocimetria da artéria umbilical e da artéria cerebral média.

\begin{tabular}{|c|c|c|c|c|c|c|}
\hline \multirow[b]{2}{*}{ Resultados perinatais } & \multicolumn{3}{|c|}{ Artéria Umbilical Normal } & \multicolumn{3}{|c|}{ Artéria Umbilical Alterada } \\
\hline & $\begin{array}{l}\text { ACM Normal } \\
(\mathrm{n}=373)\end{array}$ & $\begin{array}{c}\text { ACM Alterada } \\
\text { (Centralização } n=187 \text { ) }\end{array}$ & $p$ & $\begin{array}{l}\text { ACM Normal } \\
(n=52)\end{array}$ & $\begin{array}{c}\text { ACM Alterada } \\
\text { (Centralização } n=105 \text { ) }\end{array}$ & $\mathrm{p}$ \\
\hline $\begin{array}{l}\text { Idade gestacional no parto, } \\
\text { em semanas, média (DP) }\end{array}$ & $38,18(2,4)$ & $38,15(2,2)$ & NS & $36,09(3,2)$ & $34,37(3,5)$ & $<0,01$ \\
\hline $\begin{array}{l}\text { Peso do recém-nascido, } \\
\text { em gramas, média (DP) }\end{array}$ & $2887,8(646,0)$ & $2785,7(632,6)$ & NS & $2083,3(690,6)$ & $1810,5(769,3)$ & $<0,05$ \\
\hline Recém-nascidos PIG, n (\%) & $61(16,4 \%)$ & $41(21,9 \%)$ & NS & $30(57,7 \%)$ & $48(45,7 \%)$ & NS \\
\hline Apgar de $10 \min <7, \mathrm{n}(\%)$ & $50(13,4 \%)$ & $22(11,8 \%)$ & NS & $12(23,1 \%)$ & $46(43,8 \%)$ & $<0,05$ \\
\hline Apgar de $5 \circ \min <7, \mathrm{n}(\%)$ & $7(1,9 \%)$ & $3(1,6 \%)$ & NS & $1(1,9 \%)$ & $13(12,4 \%)$ & $<0,05$ \\
\hline $\begin{array}{l}\text { pH da artéria umbilical no } \\
\text { nascimento, média (DP) }\end{array}$ & $7,26(0,087)$ & $7,25(0,095)$ & NS & $7,25(0,084)$ & $7,20(0,114)$ & $<0,05$ \\
\hline $\begin{array}{l}\text { Acidose no nascimento }(\mathrm{pH}<7,20) \text {, } \\
\mathrm{N} / \text { total analisado }(\%)\end{array}$ & $47 / 263(17,9 \%)$ & $26 / 125(20,8 \%)$ & NS & $6 / 31(19,4 \%)$ & $31 / 80(38,8 \%)$ & NS \\
\hline $\begin{array}{l}\text { Óbito do recém-nascido no } \\
\text { berçário, } n(\%)\end{array}$ & $4(1,1 \%)$ & $1(0,5 \%)$ & NS & $1(1,9 \%)$ & $10(9,5 \%)$ & NS \\
\hline
\end{tabular}

$\mathrm{DP}=$ Desvio-padrão, NS = não-significativo, $\mathrm{ACM}=$ artéria cerebral média, $\mathrm{PIG}=$ pequeno para a idade gestacional.

\section{Discussão}

O diagnóstico precoce das alterações da vitalidade fetal reduz a ocorrência de resultados perinatais adversos porque facilita a ação oportuna do obstetra, permitindo a prevenção de lesões neurológicas decorrentes da hipoxemia e acidemia fetais. Porém, persistem as desvantagens da realização do parto prematuro intempestivo, no qual, ao se interromper uma gestação precocemente objetivando evitar as lesões neurológicas fetais da hipoxia, incorre-se no risco da ocorrência de agravos decorrentes da imaturidade fetal ${ }^{15}$, além de reduzir as chances de se obter um parto por via vaginal. Assim, o obstetra deve realizar sempre uma análise criteriosa dos resultados obtidos nos exames antenatais, orientando a conduta para proporcionar melhor resultado para o binômio mãe-filho.

Em gestações próximas ao termo, a decisão para a conduta a ser adotada é efetuada com maior facilidade, porém, quando longe do termo, os riscos decorrentes da imaturidade pulmonar influenciam constantemente as medidas a serem adotadas.

As alterações na dopplervelocimetria da AU refletem aumento de resistência ao fluxo sangüineo no território placentário, característico de anormalidade na circulação feto- placentária ${ }^{16}$. Nesta situação de falência circulatória há prejuízo nas trocas materno-fetais, culminando com a ocorrência de resultados neonatais adversos ${ }^{17,18}$. No presente estudo, podemos notar maior incidência de RN com indices de Apgar de $1^{\circ}$ e $5^{\circ}$ minutos inferior a 7 e maior proporção de óbitos neonatais no grupo com exame anormal da $\mathrm{AU}$, confirmando maior risco para estas gestações.

Podemos observar também que o diagnóstico de insuficiência placentária pela dopplervelocimetria das artérias umbilicais alteradas, quando associado ao diagnóstico de centralização fetal, apresenta pior prognóstico perinatal. Arduini e Rizzo $^{19}$, estudando 120 casos de restrição do crescimento fetal previamente avaliados pela dopplervelocimetria, observaram que a análise da artéria cerebral média apresenta os melhores valores de predição para resultados perinatais adversos. Evidentemente, a hipoxemia é decorrente do déficit da circulação placentária, proporcionando maior risco a esta gestação. Nestas situações, atualmente, realizamos avaliação rigorosa e minuciosa da circulação fetal, principalmente por meio da dopplervelocimetria do sistema venoso, que permite identificar parâmetros indicativos de risco para a acidose fetal ${ }^{20,21}$.

Alguns estudos relatam a utilização de relações entre os resultados da dopplervelocimetria da $\mathrm{ACM}$ e da AU na predição do comprometimento 
fetal $^{22,23}$. Em nosso setor utilizamos, para o diagnóstico de centralização, o valor do índice de pulsatilidade da ACM, comparando o seu resultado com os valores normais para a idade gestacional. A escolha por este tipo de análise fundamenta-se no fato de que, em situações de ausência de fluxo diastólico nas artérias umbilicais (diástole zero), as relações umbílico-cerebrais sempre serão classificadas como alteradas. Porém, temos observado que nem sempre o feto apresenta-se em situação de centralização da circulação, principalmente nas fases iniciais do comprometimento da função placentária. Outro fato importante, constatado nos casos de diástole zero, é que esta anormalidade acomete freqüentemente gestações longe do termo, situação em que a prematuridade é fator limitante na conduta a ser adotada.

Em gestações que cursam com insuficiência placentária, o diagnóstico de centralização realmente alerta quanto à possibilidade do comprometimento da oxigenação fetal, porém o intervalo até a ocorrência deste último fato é variável e deve sempre ser ponderado. Este estudo permitiu demonstrar que em gestações com função placentária normal, isto é, com dopplervelocimetria normal das AU normais, não foram constatadas diferenças nos resultados perinatais. Este fato ocorre porque o tempo entre a centralização fetal e o desenvolvimento de hipoxia fetal pode ser relativamente longo, de tal modo que outros parâmetros de avaliação da vitalidade fetal devem ser utilizados para melhor discriminação dos casos que realmente apresentem maior risco de sofrimento fetal.

Em nosso serviço, consideramos como padrão para o diagnóstico de hipoxia fetal o valor do $\mathrm{pH}$ da AU no nascimento inferior a 7,20 (acidemia fetal) ${ }^{24}$. Observamos que, no presente trabalho, não se constatou correlação entre a centralização fetal e a acidose no nascimento, mesmo em gestações com fluxo alterado nas AU, apesar de a média ter se mostrado significativamente menor no grupo com centralização.

$\mathrm{Na}$ análise da distribuição dos resultados dos testes de vitalidade fetal, observamos maior incidência de exames alterados entre os casos com centralização. As anormalidades na CTG anteparto de repouso associam-se precocemente à hipoxemia fetal, podendo justificar a correlação observada com a centralização fetal ${ }^{25}$.

Na Clínica Obstétrica da FMUSP, a proximidade do Setor de Vitalidade Fetal junto ao prénatalista especializado no controle de gestantes de alto risco permite aguardar com segurança que a gestação siga avante pelo estudo criterioso da circulação fetal e de seus mecanismos adaptativos, antes de se optar pela resolução prematura da gestação. Assim, ao serem identificados os casos de maior risco de um resultado perinatal desfavorável pela ocorrência da centralização fetal, indicamos a vigilância rigorosa, procurando reconhecer os casos que realmente se beneficiarão da resolução imediata da gestação, por meio da análise de outros parâmetros de vitalidade fetal. Percebe-se, portanto, que há longa data trabalhamos em total concordância com o que prescreveu recentemente o ACOG ${ }^{9}$.

O presente estudo demonstra a importância da aplicação de uma propedêutica abrangente do bem-estar fetal. O diagnóstico das alterações hemodinâmicas fetais é significativo apenas em gestações que cursam com algum grau de insuficiência placentária (Doppler umbilical alterado). O diagnóstico da centralização da circulação fetal pela dopplervelocimetria da ACM não influi nos resultados perinatais em gestantes com função placentária normal (Doppler umbilical normal).

\section{SUMMARY}

Purpose: to study, in high risk pregnancies with cerebral redistribution of blood flow, the fetal surveillance and perinatal outcome, according to umbilical artery dopplervelocimetry.

Methods: a total of 717 high-risk pregnancies attended at the Fetal Surveillance Unit were included. The last examination performed until $72 \mathrm{~h}$ prior to delivery was taken into account. Multiple gestations and fetal anomalies were excluded. The redistribution of blood flow was diagnosed if the pulsatility index of middle cerebral artery was below the 5 th percentile for gestational age. The umbilical artery dopplervelocimetry was abnormal when $A / B$ ratio was more than the 95 th $p$.

Results: in the group with normal umbilical artery dopplervelocimetry (560 cases - 78.1\%), significant correlation was found only between redistribution of blood flow and suspected or abnormal cardiotocography (17.1\%). In the group with abnormal umbilical artery dopplervelocimetry (157 cases $-21.9 \%$ ) we found significant correlation between redistribution of blood flow (105 cases - 66.9\%) and cardiotocography abnormalities (57.2\%), abnormal 1st (43.8\%) and 5th (12.4\%) minute Apgar scores. In these cases, the mean values of gestational age at delivery (34.4 \pm 3.6 weeks), birth weight $(1,810.5 \pm 769.3 \mathrm{~g})$, and $\mathrm{pH}$ at birth (7.20 \pm 0.1$)$ were significantly lower.

Conclusion: The redistribution of fetal blood flow characterized by means of middle cerebral artery dopplervelocimetry is related to perinatal results when some level of placental insufficiency occurs, and does not present association to perinatal outcome when pregnancy shows normal fetal-placental blood flow.

KEY WORDS: Dopplervelocimetry. Fetal outcome. Fetal surveillance. 


\section{Referências}

1. Baschat AA, Weiner CP. Umbilical artery doppler screening for detection of the small fetus in need of antepartum surveillance. Am J Obstet Gynecol 2000; 182:154-8.

2. Haley J, Tuffnell DJ, Johnson N. Randomised controlled trial of cardiotocography versus umbilical artery Doppler in the management of small for gestational age fetuses. $\mathrm{Br} \mathrm{J}$ Obstet Gynaecol 1997; 104:431-5.

3. Peeters LL, Sheldon RE, Jones MD Jr, Makowsky EL, Meschia G. Blood flow to fetal organs as a function of arterial oxygen content. Am J Obstet Gynecol 1979; 135:637-46.

4. Iwamoto HS, Kaufman T, Keil LC, Rudolph AM. Responses to acute hypoxemia in fetal sheep at 0.6-0.7 gestation. J Physiol 1989; 256:H613-20.

5. Behrman RE, Lees MH, Peterson EN, De Lannoy $\mathrm{CW}$, Seeds AE. Distribution of the circulation in the normal and asphyxiated fetal primate. Am J Obstet Gynecol 1970; 108:956-69.

6. Arduini D, Rizzo G, Romanini C. Changes of pulsatility index from fetal vessels preceding the onset of late decelerations in growth-retarded fetuses. Obstet Gynecol 1992; 79:605-10.

7. Hershkovitz R, Kingdom JC, Geary M, Rodeck CH. Fetal cerebral blood flow redistribution in late gestation: identification of compromise in small fetuses with normal umbilical artery Doppler. Ultrasound Obstet Gynecol 2000; 15:209-12.

8. Mimica M, Pejkovic L, Furlan I, Vulic Mladinic D, Praprotnik T. Middle cerebral artery velocity waveforms in fetuses with absent umbilical artery end-diastolic flow. Biol Neonate 1995; $67: 21-5$.

9. ACOG. Antepartum fetal surveillance. Practice Bulletin, number 9, October 1999. Clinical management guidelines for obstetriciangynecologists. Int $\mathrm{J}$ Gynaecol Obstet 2000; 68:175-85.

10.Amim Júnior J, Lima MLA, Fonseca ALA, Chaves Netto H, Montenegro CAB. Dopplerfluxometria da artéria umbilical: valores normais para a relação $A / B$, indice de resistência e índice pulsátil. J Bras Ginecol 1990; 100:337-49.

11.Arduini D, Rizzo G. Normal values of pulsatility index from fetal vessels: a cross sectional study on 1556 healthy fetuses. J Perinat Med 1990; 18:165-72.

12.Zugaib M, Behle I. Monitoração fetal eletrônica. $1^{\mathrm{a}}$ ed. São Paulo: Roca; 1981. p.55-78.

13. Phelan JP, Smith CV, Broussard P, Small M. Amniotic fluid volume assessment with the fourquadrant technique at 36-42 weeks' gestation. J Reprod Med 1987; 32:540-2.
14. Ramos JLA. Avaliação do crescimento intra-uterino por medidas antropométricas do recém-nascido [tese]. São Paulo: Universidade de São Paulo; 1983.

15.Black RS, Campbell S. Cardiotocography versus Doppler. Ultrasound Obstet Gynecol 1997; 9:14851.

16.Trudinger BJ, Giles WB, Cook CM, Bombardieri J, Collins L. Fetal umbilical artery flow velocity waveforms and placental resistance: clinical significance. Br J Obstet Gynaecol 1985; 92:2330.

17.Almstrom $\mathrm{H}$, Axelsson $\mathrm{O}$, Cnattingius $\mathrm{S}$, et al. Comparison of umbilical-artery velocimetry and cardiotocography for surveillance of small-forgestational-age fetuses. Lancet 1992; 340:93640.

18.Nienhuis SJ, Vles JS, Gerver WJ, Hoogland HJ. Doppler ultrasonography in suspected intrauterine growth retardation: a randomized clinical trial. Ultrasound Obstet Gynecol 1997; 9:6-13.

19.Arduini D, Rizzo G. Prediction of fetal outcome in small for gestational age fetuses: comparison of Doppler measurements obtained from different fetal vessels. J Perinat Med 1992; 20:29-38.

20.Francisco RPV. Predição da acidose no nascimento em gestações com diástole zero ou reversa à dopplervelocimetria das artérias umbilicais [dissertação]. São Paulo: Universidade de São Paulo; 1998.

21.Hecher K, Hackelöer BJ. Cardiotocogram compared to Doppler investigation of the fetal circulation in the premature growth-retarded fetus: longitudinal observations. Ultrasound Obstet Gynecol 1997; 9:152-61.

22. Ott WJ. Comparison of the non-stress test and middle cerebral to umbilical artery systolic/ diastolic velocity wave form ratio for the prediction of neonatal compromise. J Matern Fetal Invest 1996; 6:73-6.

23.Gramellini D, Folli MC, Raboni S, Vadora E, Merialdi A. Cerebral-umbilical Doppler ratio as a predictor of adverse perinatal outcome. Obstet Gynecol 1992; 79:416-20.

24.Francisco RPV, Yamamoto RM, Miyadahira S, Cunha CL, Zugaib M. Correlação entre testes para avaliação da vitalidade fetal, $\mathrm{pH}$ da artéria umbilical e os resultados neonatais em gestações de alto risco. Rev Bras Ginecol Obstet 2000; 22:503-10.

25.Visser GH, Sadovsky G, Nicolaides KH. Antepartum heart rate patterns in small-for-gestational-age third-trimester fetuses: correlations with blood gas values obtained at cordocentesis. Am J Obstet Gynecol 1990; 162:698-703. 